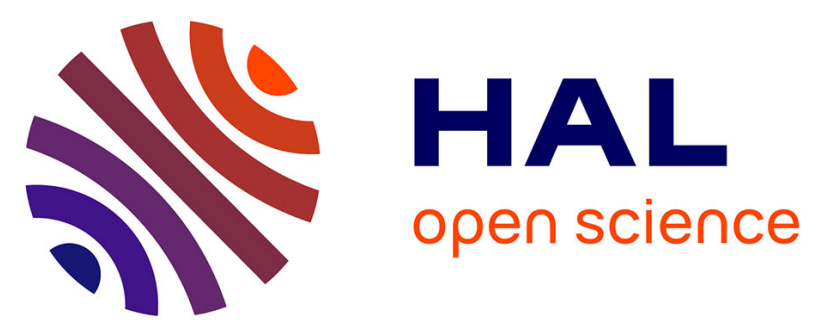

\title{
Troubles neuropsychiatriques inexpliqués : penser aux encéphalites dysimmunitaires. À propos d'une observation d'encéphalite à anticorps anti-leucine rich glioma inactivated 1 (LGI-1)
}

E. Le Dault, Stanislas Lagarde, Eric Guedj, B. Dufournet, C. Rey, E. Kaphan, G. Tanguy, M. Bregigeon, E. Sagui, C. Brosset

\section{To cite this version:}

E. Le Dault, Stanislas Lagarde, Eric Guedj, B. Dufournet, C. Rey, et al.. Troubles neuropsychiatriques inexpliqués : penser aux encéphalites dysimmunitaires. À propos d'une observation d'encéphalite à anticorps anti-leucine rich glioma inactivated 1 (LGI-1). La Revue de Médecine Interne, 2016, 37 (2), pp.127-130. 10.1016/j.revmed.2015.06.007 . hal-01851669

\author{
HAL Id: hal-01851669 \\ https://hal.science/hal-01851669
}

Submitted on 21 Mar 2020

HAL is a multi-disciplinary open access archive for the deposit and dissemination of scientific research documents, whether they are published or not. The documents may come from teaching and research institutions in France or abroad, or from public or private research centers.
L'archive ouverte pluridisciplinaire HAL, est destinée au dépôt et à la diffusion de documents scientifiques de niveau recherche, publiés ou non, émanant des établissements d'enseignement et de recherche français ou étrangers, des laboratoires publics ou privés. 


\title{
Communication brève
}

\section{Troubles neuropsychiatriques inexpliqués : penser aux encéphalites dysimmunitaires. À propos d'une observation d'encéphalite à anticorps anti-leucine rich glioma inactivated 1 (LGI-1)}

\section{Unexplicated neuropsychiatric disorders: Do not ignore dysimmune encephalitis.}

\section{A case report of a dysimmune encephalitis with anti-leucine rich glioma} inactivated 1 (LGI-1) antibodies

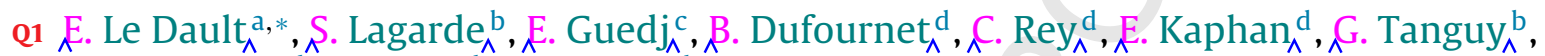 \\ M. Bregigeon ${ }_{\wedge}^{\mathrm{b}}, \mathrm{E}$. Sagui ${ }_{\wedge}^{\mathrm{b}},{ }_{\wedge}$. . Brosset ${ }_{\wedge}^{\mathrm{b}}$ \\ a Service de pathologie infectieuse et tropicale, hôpital d'instruction des armées Laveran, 34, boulevard Alphonse-Laveran, 13014 Marseille, France \\ b Service de neurologie, hôpital d'instruction des armées Laveran, 34, boulevard Alphonse-Laveran, 13014 Marseille, France \\ ' Service de médecine nucléaire, CHU La Timone, AP-HM, 264, rue Saint-Pierre, 13385 Marseille, France \\ d Service de neurologie, CHU La Timone, AP-HM, 264, rue Saint-Pierre, 13385 Marseille, France
}

I N F O A R T I C L E

\section{Historique de l'article :}

Disponible sur Internet le xxx

\section{Mots clés :}

Encéphalite dysimmunitaire Encéphalite auto-immune Leucine rich glioma inactivated 1 (LGI-1) Voltage gate potassium channel (VGKC) Spasmes brachio-faciaux

\begin{abstract}
R É S U M É
Introduction. - Diagnostiquer une encéphalite dysimmunitaire est difficile bien qu'il s'agisse de pathologies non rares, de mieux en mieux décrites. Le bilan étiologique de première intention de ces encéphalites reste souvent normal et peu informatif.

Observation. - Nous rapportons l'observation d'un patient de 48 ans qui présentait des troubles cognitifs et comportementaux, des mouvements anormaux (spasmes brachio-faciaux), des crises d'épilepsie et un syndrome de sécrétion inappropriée d'hormone antidiurétique. Après élimination d'une encéphalite infectieuse, néoplasique, inflammatoire systémique, métabolique ou toxique, la tomographie par émission de positons cérébrale au ${ }^{18}$ Fluoro-désoxy-glucose $\left({ }^{18} \mathrm{FDG}\right)$ et la recherche d'anticorps antineuronaux dans le liquide céphalorachidien et le sérum permettaient de diagnostiquer une encéphalite dysimmunitaire à anticorps anti-canaux potassiques voltage-dépendants de type anti-leucine rich glioma inactivated 1. L'évolution était favorable sous cyclophosphamide après une efficacité incomplète des immunoglobulines intraveineuses et de la corticothérapie systémique.

Conclusion. - Il faut savoir évoquer une encéphalite dysimmunitaire devant un tableau neuropsychiatrique aigu à bilan étiologique négatif. Les signes évocateurs d'une encéphalite à anti-leucine rich glioma inactivated 1 sont les spasmes brachio-faciaux et le syndrome de sécrétion inappropriée d'hormone antidiurétique. Son diagnostic repose sur des examens ciblés (tomographie par émission de positons cérébrale au ${ }^{18}$ FDG et recherche d'anticorps dans le liquide céphalorachidien et le sérum). Un bilan exhaustif et répété à la recherche d'une néoplasie occulte est nécessaire. Les traitements immunosuppresseurs sont
\end{abstract} efficaces.

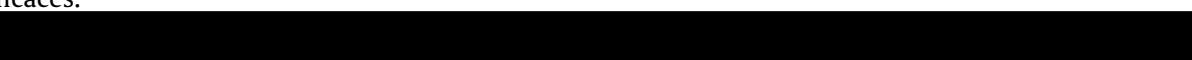

\section{A B S T R A C T}

Introduction. - Anti-leucine rich glioma inactivated 1 encephalitis is a common and a treatable etiology of autoimmune encephalitis. Its diagnosis is a challenge because the initial diagnostic work-up is often normal.

Case report. - A 48-year-old man experienced cognitive and behavioral troubles, facio-brachial dystonic seizures and a syndrome of inappropriate antidiuretic hormone secretion. First line tests excluded infectious, neoplastic, systemic inflammatory, endrocrine or toxic etiologies. Cerebral

* Auteur correspondant.

Adresse e-mail : erwan.le.dault@gmail.com (E. Le Dault). 
${ }^{18}$ Fluoro-desoxy-glucose (FDG) position emission tomography and research of specific antibodies in cerebro-spinal fluid and serum led to diagnose an anti-leucine rich glioma inactivated 1 encephalitis. Intravenous immunoglobulins and corticosteroids were partially effective. Cyclophosphamid permitted a good recovery.

Conclusion. - In the presence of acute neuropsychiatric disorders with a negative etiologic research, physician should think about dysimmune encephalitis. Facio-brachial dystonic seizures and syndrome of inappropriate antidiuretic hormone secretion are highly evocative of anti-leucine rich glioma inactivated 1 encephalitis. The diagnosis needs specific diagnostic tests (cerebral ${ }^{18}$ FDG position emission tomography and antibodies research in cerebro-spinal fluid and in serum), after the exclusion of alternative diagnoses. Extensive and repeated diagnostic work-up for neoplasia is required. Immunosupressive therapies are effective in most cases.

(c) 2015 Published by Elsevier Masson SAS on behalf of the Société nationale française de médecine interne (SNFMI)

\section{1. Introduction}

\section{Observation} quantel.
Les encéphalites dysimmunitaires sont un groupe hétérogène de pathologies associées à des anticorps ciblant des antigènes membranaires ou intracellulaires du système nerveux central [1]. Malgré une connaissance de leur spectre plus précise, elles restent sous diagnostiquées. Leurs manifestations cliniques sont sévères et handicapantes, mais aspécifiques. Les examens de première intention (biologie sanguine, imagerie par résonance magnétique [IRM] cérébrale, électroencéphalogramme [EEG] et analyse du liquide céphalorachidien [LCR]) manquent de sensibilité et de spécificité. Ils permettent d'éliminer les diagnostics différentiels infectieux, néoplasiques, métaboliques, inflammatoires systémiques, qui doivent rester le premier réflexe du clinicien. Leur diagnostic positif nécessite une démarche rigoureuse avec des examens ciblés (tomographie par émission de positons au ${ }^{18}$ Fluorodésoxy-glucose [ ${ }^{18} \mathrm{FDG}$-TEP] cérébrale et recherche d'anticorps spécifiques dans le LCR ou le sérum).

Nous rapportons l'observation d'un patient chez lequel l'association de troubles cognitifs, de troubles comportementaux, de mouvements anormaux et de crises d'épilepsie révélait une encéphalite à anticorps anti-membranaires de type anti-leucine rich glioma inactivated 1 (LGI-1). Nous discuterons notre démarche diagnostique face à une suspicion d'encéphalite dysimmunitaire, puis exposerons les aspects cliniques, paracliniques et thérapeutiques des encéphalites à anticorps anti-LGI-1.

Un homme de 48 ans, ancien parachutiste, avait comme pathologies évolutives un tabagisme actif et des troubles dysthymiques (traités par carbamazépine et paroxétine). Il présentait un antécédent d'accident de la voie publique avec traumatisme crânien et un épisode d'hyperéosinophilie résolutif après traitement probabiliste antiparasitaire par albendazole, ivermectine et prazi-

La découverte d'un nodule pulmonaire hyper-métabolique en TEP, conduisait à réaliser une lobectomie inférieure droite curative. L'examen histologique retrouvait un granulome aspécifique sans nécrose caséeuse. Le bilan d'extension (tomodensitométrie [TDM] thoraco-abdomino-pelvienne), TEP-TDM corps entier au ${ }^{18} \mathrm{FDG}$, scintigraphie osseuse, échographie testiculaire et dosage des marqueurs tumoraux sériques (AFP, CA 15-3, CA 125, CA 19-9, ACE, HCG, CYFRA 21, NSE, PSA, $\beta 2$ microglobuline, LDH) était négatif. Les suites opératoires se compliquaient d'une pleurésie enkystée à Streptococcus mitis et de multiples fractures ostéoporotiques spontanées de T4 à T12. L'infection du site opératoire guérissait après drainage chirurgical et antibiothérapie adaptée.

Parallèlement, le patient présentait des troubles cognitifs (amnésie antérograde, anosognosie, lenteur idéomotrice) et comportementaux (désinhibition, persévération, écholalie, akathisie) fluctuants et rapidement progressifs. L'examen clinique objectivait des spasmes brachio-faciaux à bascule. Il n'existait pas d'aphtose, d'arthrite, d'anomalie cutanée, d'adénopathie, de syndrome sec ou de sérite.

Les imageries cérébrales morphologiques (TDM et IRM cérébrales injectées avec séquences vasculaires) éliminaient un accident vasculaire cérébral ischémique ou hémorragique, une vascularite cérébrale, une thrombose veineuse cérébrale, une pathologie inflammatoire démyélinisante, un processus expansif intracrânien, une encéphalite nécrosante ou une pathologie neurodégénérative évoluée. Il n'existait en particulier ni anomalie des lobes temporaux ni atrophie cérébrale. L'électroencéphalogramme (EEG) montrait un rythme de fond normal et des bouffées d'ondes lentes bi-frontales. L'analyse du liquide cérébro-rachidien (LCR) retrouvait une hyperprotéinorachie minime à $0,57 \mathrm{~g} / \mathrm{L}$, une normoglycorachie, une cellularité normale, une absence de synthèse intrathécale d'immunoglobulines, des cultures bactériennes et mycologiques stériles et des PCR pour les virus neurotropes (HSV, VZV, entérovirus) négatives. Le bilan biologique révélait une hyponatrémie à $127 \mathrm{mmol} / \mathrm{L}$, hypo-osmolaire, à natriurèse conservée compatible avec un syndrome de sécrétion inappropriée d'hormone antidiurétique (SIADH). Il n'y avait pas de carence vitaminique B1, B6, B9 ou B12. Les sérologies VIH, syphilis, hépatites B et $C$, maladie de Lyme, rickettsiose, fièvre $Q$ bartonellose étaient négatives.

La négativité des explorations précédentes et l'atteinte pseudo-systémique (granulome pulmonaire, hyperéosinophilie à l'anamnèse, pleurésie, fractures vertébrales) faisaient discuter une pathologie inflammatoire systémique avec atteinte cérébrale secondaire : lupus, maladie de Behçet, sarcoïdose, vascularite, syndrome des anti-phospholipides. Le bilan biologique ne retrouvait pas d'auto-anticorps anti-nucléaires, anti-cytoplasme des polynucléaires neutrophiles, anti-phospholipides, antithyroïdiens ou d'anomalies du dosage de l'enzyme de conversion de l'angiotensine. La biopsie des glandes salivaires accessoires ne retrouvait pas de sialadénite ou de granulome.

Compte tenu des signes encéphalitiques (troubles cognitifs, comportementaux, épilepsie) et en l'absence de diagnostic étiologique, une encéphalite dysimmunitaire était suspectée. La TEP cérébrale au ${ }^{18}$ FDG (Fig. 1) confirmait le diagnostic d'encéphalite, montrant un hyper-métabolisme bistriatal et mésio-temporal droit, associé à un hypo-métabolisme cortical diffus. La présence de spasmes brachio-faciaux et d'un SIADH orientait vers une encéphalite à anticorps anti-LGI-1. La positivité des anticorps dans le LCR et le sang confirmait cette étiologie: dosage radio-immunologique des anticorps anticanaux potassiques voltage-dépendants (VGKC) dans le sérum à $448 \mathrm{pmol} / \mathrm{L}(\mathrm{N}<72 \mathrm{pmol} / \mathrm{L}$; laboratoire d'immunologie, CHU la Timone, AP-HM, Marseille); confirmé sur le LCR par le test cell based assay sur cellules HEK 293 (immunofluorescence sur des cellules surexprimant des récepteurs cibles) retrouvant une 


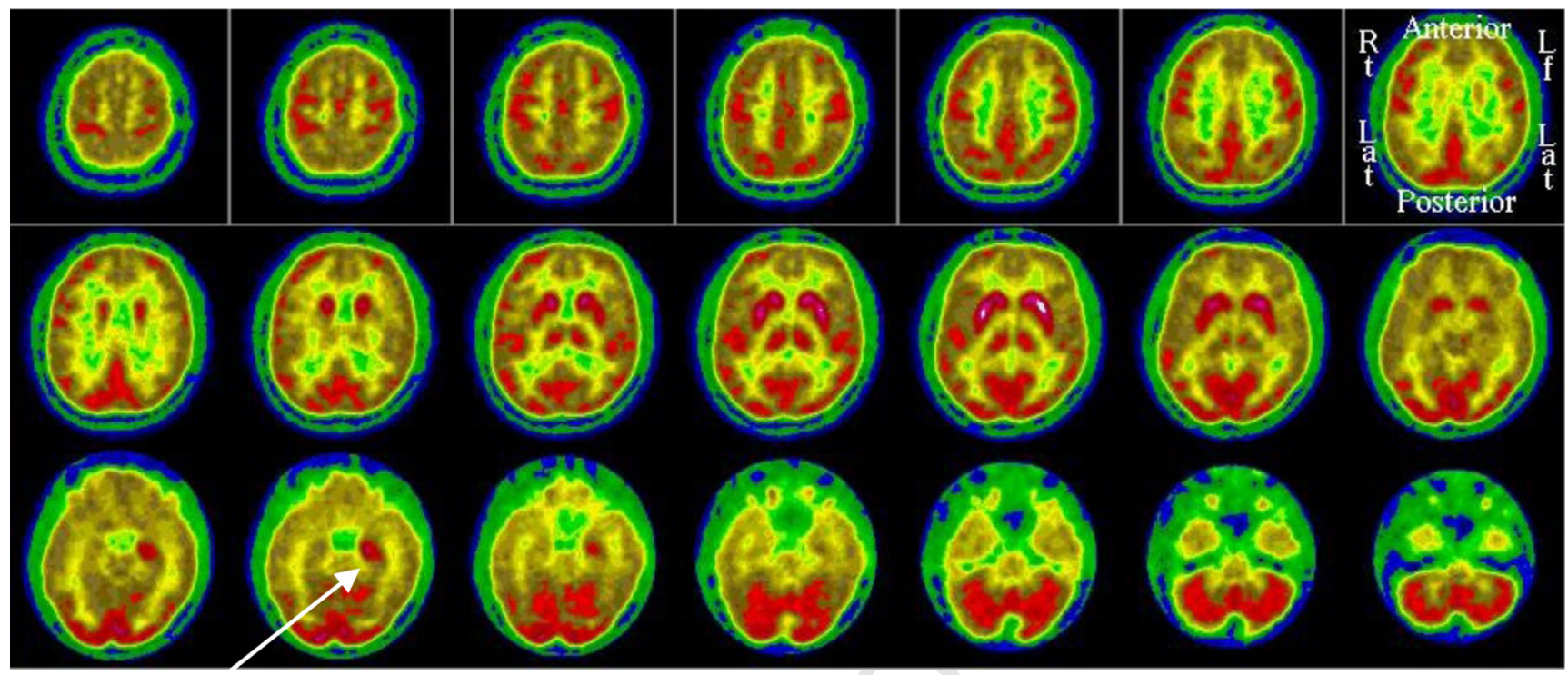

Fig. 1. ${ }^{18}$ FDG-PET cérébrale initiale.

spécificité anti-LGI-1 (Centre de référence pour les syndromes paranéoplasiques, $\mathrm{CHU}$ de Lyon $\mathrm{HCL}$, Pr Honnorat).

La corticothérapie systémique (1 cure intraveineuse de $1 \mathrm{~g}$ par jour pendant 5 jours) et les immunoglobulines intraveineuses ( 2 cures mensuelles de $2 \mathrm{~g} / \mathrm{kg}$ pendant 5 jours) s'avéraient d'efficacité incomplète. L'arrêt de l'inhibiteur spécifique de la recapture de la sérotonine (paroxétine) et l'introduction d'un traitement antiépileptique (lévétiracétam) ne permettaient respectivement ni la résolution du SIADH ni celle des spasmes brachio-faciaux.

Finalement, la mise sous cyclophosphamide $\left(750 \mathrm{mg} / \mathrm{m}^{2}\right.$ par mois, adapté selon la tolérance hématologique) s'accompagnait d'une amélioration des troubles cognitifs, comportementaux, des spasmes brachio-faciaux et du SIADH. Les anomalies métaboliques se corrigeaient partiellement sur la ${ }^{18}$ FDGTEP cérébrale de contrôle après 3 cures de cyclophosphamide (Fig. 2).

\section{Discussion}

Les encéphalites dysimmunitaires doivent être évoquées devant un tableau neuropsychiatrique aigu (amnésie et troubles comportementaux) après un premier bilan étiologique négatif $[1,2]$. Il existe deux groupes d'encéphalites dysimmunitaires qui diffèrent par leurs cibles antigéniques: intracellulaires (anticorps anti-Hu, anti-Ri, anti-Tr, anti-Yo, anti-Ma2, anti-CV2/CRMP5, anti-glutamic acid decarboxylase (GAD), anti-amphyphysine) [1-3], ou membranaires (anticorps anti-N-méthyl-D-aspartate récepteur [NMDA], anti-canaux potassiques voltage-dépendants [VGKC] incluant LGI1, Caspr2, contactin-2, anti-AMPA récepteur, anti-GABA $A_{B}$ récepteur, anti-glycine récepteur, anti-mGluR5, anti-D2R) [1,3,4]. Ces deux groupes se distinguent par leur origine paranéoplasique, leur pronostic et leur prise en charge. Les encéphalites à cibles antigéniques intracellulaires sont dans plus de $90 \%$ des cas paranéoplasiques (séminomes, cancers bronchiques à petites cellules, thymomes, cancers du sein) $[1,3,5]$. Leur prise en charge repose surtout sur celle de la néoplasie causale et leur pronostic reste défavorable [3,4]. Les encéphalites à cibles antigéniques membranaires sont moins souvent paranéoplasiques [4] et de pronostic plus favorable. Leur traitement fait appel aux immunomodulateurs ou aux immunosuppresseurs [2-4,6-8].
Les encéphalites à anti-LGI-1 font partie des encéphalites à anticorps membranaires, du complexe anti-VGKC. Les spasmes brachio-faciaux sont quasi pathognomoniques de ce type d'encéphalites [1,6-11]. Ils peuvent précéder l'apparition des troubles cognitifs, être à bascule et résistants aux antiépileptiques [9]. La présence d'un SIADH est aussi très évocatrice du diagnostic $[2,3,5,6,8,12]$. Les autres symptômes sont moins spécifiques: troubles cognitifs surtout mnésiques $[1,2,6,11]$, troubles comportementaux $[1,2,6,13,14]$, mouvements choréiques [15], crises d'épilepsie [1-3,6], dysautonomie (hypotension orthostatique, constipation, incontinence urinaire) [1,11], syndrome parkinsonien, ataxie $[1,2,16]$, somnolence et insomnie $[1,3,6]$, douleurs neuropathiques [2]. L'IRM peut être normale ou montrer un hypersignal T2/FLAIR temporal (plus ou moins étendu, parfois bilatéral ou associé à une atrophie mésio-temporale) [2,3,5,6,8,11,12]. L'analyse du LCR peut être normale ou retrouver une hyperprotéinorachie modérée, une pleïocytose ou une synthèse intrathécale d'immunoglobulines [2,3,5,6,8,11,12]. Il n'existe pas d'aspect EEG spécifique $[1,3,17,18]$. La ${ }^{18}$ FDG-TEP cérébrale est l'examen d'imagerie le plus sensible et le plus informatif. Elle montre classiquement l'association d'un hyper-métabolisme temporal ou des ganglions de la base (parfois cervelet, région occipitale et précentrale) et d'un hypo-métabolisme cortical antérieur (région cingulaire antérieure et fronto-mésiale) [1,3,11,19,20]. Finalement, la présence d'anticorps anti-LGI-1 dans le LCR et le sérum confirme le diagnostic [2].

Les encéphalites à anti-LGI-1 sont associées à une néoplasie dans moins de $10 \%$ des cas $[1-5,7,8,11]$. Cette association, bien que rare, justifie une recherche systématique, exhaustive et répétée d'un cancer (examen clinique complet en particulier des organes génitaux externes et des aires ganglionnaires, TDM thoraco-abdomino-pelvienne injectée, échographie testiculaire, voire TEP-TDM corps entier et dosage des marqueurs tumoraux).

En dehors de ces rares cas paranéoplasiques, elles répondent favorablement au traitement immunosuppresseur comme l'illustre l'observation rapportée. Le traitement de première intention des encéphalites à anti-LGI-1 repose sur l'utilisation de corticostéroïdes ( $1 \mathrm{~g} / \mathrm{j}$ intraveineux pendant 5 jours), d'immunoglobulines intraveineuses ( 3 cures mensuelles de $2 \mathrm{~g} / \mathrm{kg}$ en 5 jours), de plasmaphérèses, en monothérapie ou en association. En cas d'échec, l'utilisation du cyclophosphamide $\left(750 \mathrm{mg} / \mathrm{m}^{2} / \mathrm{mois}\right.$ 


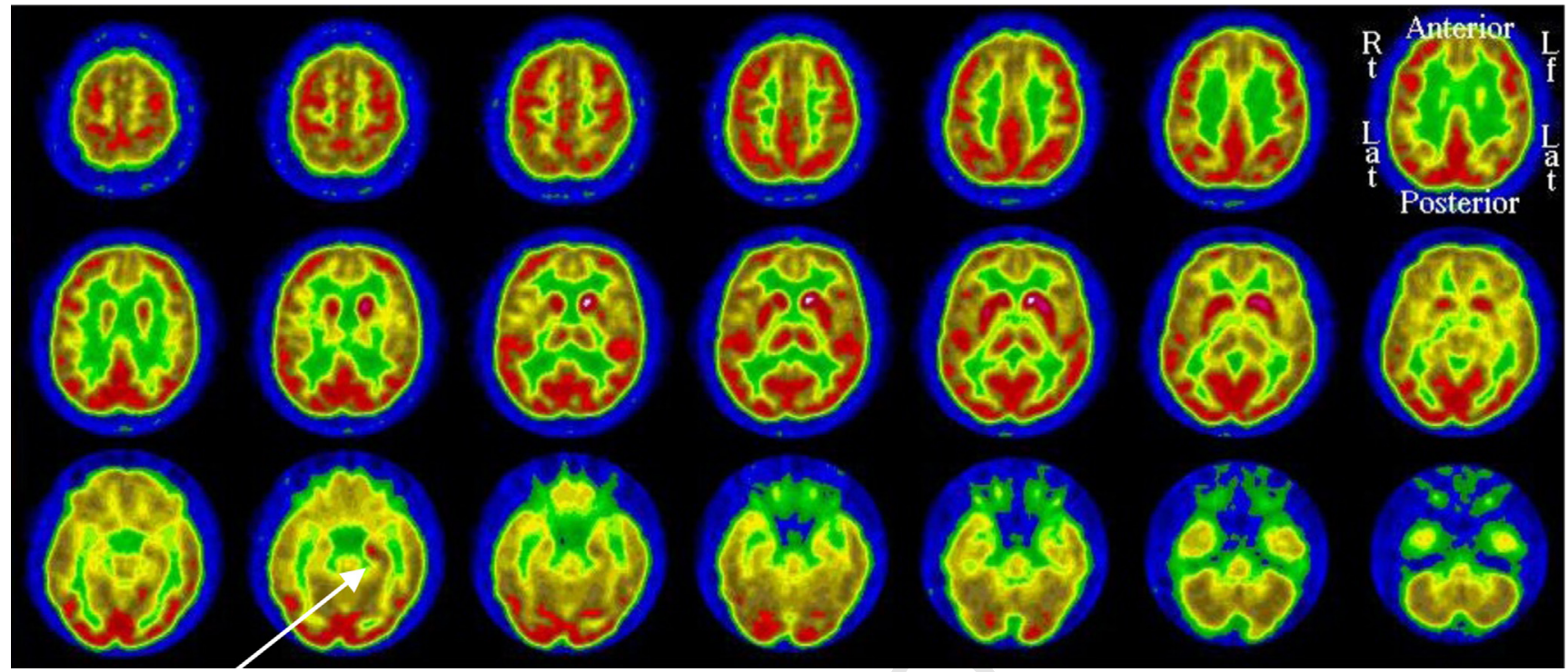

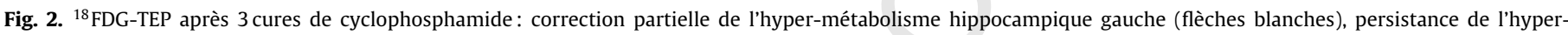
métabolisme des striati et de l'hypo-métabolisme cortical diffus notamment au niveau temporal gauche.

223 pendant 6 mois) ou du rituximab $\left(375 \mathrm{mg} / \mathrm{m}^{2} /\right.$ semaine pendant 4 semaines) a montré des résultats encourageants [1-4,6,11]. Ces stratégies thérapeutiques reposent sur des séries rétrospectives d'effectifs restreints et aucun essai randomisé n'a été jusqu'alors réalisé. La description de deux cas d'évolution spontanément favorable sans immunomodulateurs après 3 et 5 ans de suivi fait discuter la pertinence de tels traitements [21].

\section{Conclusion}

Les tableaux neuropsychiatriques aigus, surtout associés à une épilepsie ou des mouvements anormaux, doivent faire évoquer le diagnostic d'encéphalite dysimmunitaire. L'existence de crises dystoniques brachio-faciales et d'un SIADH est particulièrement évocatrice d'une encéphalite à anticorps anti-LGI-1. La réalisation d'une ${ }^{18} \mathrm{FDG}$-TEP cérébrale et la recherche d'anticorps anti-neuronaux dans le LCR et le sérum permettent de confirmer le diagnostic. L'efficacité des traitements immunomodulateurs ou immunosuppresseurs dans les encéphalites à anticorps antineuronaux à cible membranaire non paranéoplasiques en font tout l'enjeu diagnostique. Il ne faut pas négliger la rechercher d'une néoplasie sous-jacente qui peut rarement lui être associée.

\section{Déclaration d'intérêts}

Les auteurs déclarent ne pas avoir de conflits d'intérêts en relation avec cet article.

\section{Remerciements}

Nous remercions le Pr Jérôme Honnorat et son équipe pour leur aide au diagnostic immunologique et leurs conseils sur la prise en charge thérapeutique du patient.

\section{Références}

[1] Vincent A, Bien CG, Irani SR, Waters P. Autoantibodies associated with diseases of the CNS: new developments and future challenges. Lancet Neurol 2011;10:759-72.

[2] Irani SR, Gelfand JM, Al-Diwani A, Vincent A. Cell-surface central nervous system autoantibodies: clinical relevance and emerging paradigms. Ann Neurol 2014;76:168-84.
[3] Aupy J, Collongues N, Blanc F, Tranchant C, Hirsch E, De Seze J. Autoimmune encephalitis, clinical, radiological and immunological data. Rev Neurol 2013;169:142-53.

[4] Honnorat J. Therapeutic approaches in antibody-associated central nervous system pathologies. Rev Neurol 2014;170:587-94.

[5] Lai M, Huijbers MG, Lancaster E, et al. Investigation of LGI1 as the antigen in limbic encephalitis previously attributed to potassium channels: a case series. Lancet Neurol 2010;9:776-85.

[6] Irani SR, Alexander S, Waters $P$, et al. Antibodies to Kv1 potassium channel-complex proteins leucine-rich, glioma inactivated 1 protein and contactin-associated protein-2 in limbic encephalitis, Morvan's syndrome and acquired neuromyotonia. Brain 2010;133:2734-48.

[7] Irani SR, Stagg CJ, Schott JM, et al. Faciobrachial dystonic seizures: the influence of immunotherapy on seizure control and prevention of cognitive impairment in a broadening phenotype. Brain 2013;136:3151-62.

[8] Vincent A, Buckley C, Schott JM, et al. Potassium channel antibody-associated encephalopathy: a potentially immunotherapy-responsive form of limbic encephalitis. Brain 2004;127:701-12.

[9] Irani SR, Michell AW, Lang B, et al. Faciobrachial dystonic seizures precede Lgi1 antibody limbic encephalitis. Ann Neurol 2011;69:892-900.

[10] Sen A, Wang J, Laue-Gizzi H, Lee T, Ghougassian D, Somerville ER. Pathognomonic seizures in limbic encephalitis associated with anti-LGI1 antibodies. Lancet 2014;383:2018.

[11] Shin YW, Lee ST, Shin JW, et al. VGKC-complex/LGI1-antibody encephalitis: clinical manifestations and response to immunotherapy. J Neuroimmunol 2013;265:75-81.

[12] Thieben MJ, Lennon VA, Boeve BF, Aksamit AJ, Keegan M, Vernino S. Potentially reversible autoimmune limbic encephalitis with neuronal potassium channel antibody. Neurology 2004;62:1177-82.

[13] Geschwind MD, Shu H, Haman A, Sejvar JJ, Miller BL. Rapidly progressive dementia. Ann Neurol 2008;64:97-108.

[14] Somers KJ, Lennon VA, Rundell JR, et al. Psychiatric manifestations of voltagegated potassium-channel complex autoimmunity. J Neuropsychiatry Clin Neurosci 2011;23:425-33.

[15] Tofaris GK, Irani SR, Cheeran BJ, Baker IW, Cader ZM, Vincent A Immunotherapy-responsive chorea as the presenting feature of LGI1-antibody encephalitis. Neurology 2012;79:195-6.

[16] Tan KM, Lennon VA, Klein CJ, Boeve BF, Pittock SJ. Clinical spectrum of voltagegated potassium channel autoimmunity. Neurology 2008;70:1883-90.

[17] Barajas RF, Collins DE, Cha S, Geschwind MD. Adult-onset drug-refractory seizure disorder associated with anti-voltage-gated potassium-channel antibody. Epilepsia 2010;51:473-7.

[18] Gast H, Schindler K, Z'Graggen WJ, Hess CW. Improvement of nonparaneoplastic voltage-gated potassium channel antibody-associated limbic encephalitis without immunosuppressive therapy. Epilepsy Behav 2010;17:555-7.

[19] Fauser S, Talazko J, Wagner K, et al. FDG-PET and MRI in potassium channe antibody-associated non-paraneoplastic limbic encephalitis: correlation with clinical course and neuropsychology. Acta Neurol Scand 2005;111:338-43.

[20] Wegner F, Wilke F, Raab P, et al. Anti-leucine rich glioma inactivated 1 protein and anti-N-methyl-D-aspartate receptor encephalitis show distinct patterns of brain glucose metabolism in ${ }^{18} \mathrm{~F}$-fluoro-2-deoxy-d-glucose positron emission tomography. BMC Neurol 2014;14:136.

[21] Szots M, Marton A, Kover F, et al. Natural course of LGI1 encephalitis: 3-5 years of follow-up without immunotherapy. J Neurol Sci 2014;343:198-202. 